Известия НАН Армении, Физика, т.57, №1, с.30-43 (2022)

УДК 53.082.6, 533.6.011, 534.11

DOI: $10.54503 / 0002-3035-2022-57.1-30$

\title{
ГАЗОВЫЙ ПОТОКОМЕР НА ОСНОВЕ ВИБРИРУЮЩИХ СТРУН
}

\author{
М.А. АГИНЯН ${ }^{1}$, С.Г. АРУТЮНЯН ${ }^{1}$, Г.С. АРУТЮНЯН ${ }^{1}$, Е.Э. ГУКАСЯН ${ }^{1}$, \\ Э.Г. ЛАЗАРЕВА ${ }^{1 *}$, А.В. МАРГАРЯН ${ }^{1}$, Л.А. ПОГОСЯН ${ }^{1}$, \\ M. $\mathrm{CHUNG}^{2}$, D. KWAK ${ }^{2}$, R. REETZ ${ }^{3}$ \\ ${ }^{1}$ Национальная научная лаборатория им. А.И. Алиханяна, Ереван, Армения \\ ${ }^{2}$ Ulsan National Institute of Science and Technology, Ulsan, South Korea \\ ${ }^{3}$ HTM-Reetz GmbH, Berlin, Germany \\ *e-mail: ella.lazareva@yerphi.am \\ (Поступила в редакцию 9 сентября 2021 г.)
}

\begin{abstract}
В работе предложен новый вид газовых потокомеров, основанный на измерениях деформаций теплового поля нагревателя в присутствии потока с помощью вибрирующих струн. Преимуществом таких потокомеров по сравнению с известными, использующими аналогичный тепловой принцип действия, является улучшенная точность измерений температуры по сравнению с резистивными термометрами или термопарами. Использование тонких струн позволяет также увеличить быстродействие потокомеров. Использование струн длиной 20-30 мм позволяет создавать широкоапертурные приемные отверстия для газовых потоков. Естественным свойством разработанных потокомеров является также их двунаправленность. Изготовлен образец потокомера на базе вибрирующих струн и проведены калибровочные эксперименты. Проведен эксперимент, в котором потокомер использовался для измерения ускорения.
\end{abstract}

\section{1. Введение}

Газовые потокомеры широко используются во многих областях науки и техники. Измерение и разделение потоков газа играют важную роль в химической, фармацевтической, металлургической и аэрокосмической промышленностях, производстве медицинских препаратов, керамике и во многих технологических процессах. При изготовлении полупроводников измерения потоков газа и их дозирование имеет также важное значение, например, для организации процессов осаждения плазмы. Точные измерители расхода воздуха используются для оптимизации процессов сгорания топлива в двигателях автомобилей.

Измерение потоков газа актуально для высокотемпературных печей: обдув камер нагревательных элементов защитными газами, измерение выхлопных газов, образующихся в нагревательных камерах при термохимических процессах (выхлопные газы могут быть агрессивными, кислотными или щелочными, содержать водяной пар и пыль). 
Особая сфера применения потокомеров - медицина, где эти приборы используются для диагностики определенных заболеваний, связанных с измерениями дыхания пациента. Эти измерения позволяют судить о наличии и степени тяжести заболевания (например, в случае астмы и COVID-19). Потокомеры являются неотъемлемой частью устройств искусственной вентиляции легких при использовании кислорода и других газов (например, гелия, окиси азота).

На сегодняшний день существует множество методов измерения и дозирования потоков газов: кориолисовые, ультразвуковые, анемометрические, электромагнитные, переменного перепада давления, турбинные, тепловые [1]. Так, в кориолисовых потокомерах измеряется сила Кориолиса, возникающая при движении потока газа по изогнутой линии; ультразвуковые потокомеры основаны на изменении скорости звука в движущейся среде; вихревые потокомеры основаны на измерениях завихрений за телом, обтекаемым потоком; тепловые, анемометрические потокомеры основаны на измерениях искажений теплового поля, вызванных потоками газа; в основе электромагнитных потокомеров лежит принцип электромагнитной индукции; в турбинных потокомерах поток вращает турбину, скорость вращений которой измеряется и служит показателем величины потока.

В науке и технике используется прецизионный класс потокомеров, основанный на измерении температурного градиента в газе от локального источника тепла. Принцип работы заключается в измерении этих градиентов в зависимости от величины расхода. Для измерения смещения теплового поля используется следующая процедура: поток газа точно делится специальным устройством в известной пропорции (обычно 1:100 и 1:1000), и эта небольшая часть потока проводится через тонкий капилляр. В центральной части капилляра располагается нагревательный провод, намотанный на капилляр и два резистивных термометра в виде дополнительных обмоток на капилляре по обе стороны от нагревательной обмотки. В связи с особенностями процесса передачи тепла от газа к термометру реальный вклад вносят массовые потоки газов, поэтому потокомеры данного типа обычно называют массовыми потокомерами. Класс таких потокомеров является наиболее точным среди существующих, однако предъявляет значительные требования к точности изготовления всех деталей датчиков. Кроме того, газ должен находиться под давлением (обычно в несколько атмосфер), чтобы измеряемое количество газа проходило через капилляр. Еще одним недостатком капиллярных массовых потокомеров является их инерционность, обусловленная существенной теплоемкостью капилляров и термометров.

Другим классом потокомеров с большой входной апертурой и тепловым принципом работы являются так называемые анемометрические датчики, в которых аналогичная идея теплопередачи, в зависимости от продувки термометра, реализуется в трубе с достаточно большим поперечным сечением. Как правило, в качестве термометров здесь используются термисторы. Различают потокомеры с горячей и холодной струной. В случае горячей струны - струна служит и нагревателем, и термометром. В случае холодных струн эти струны разделены. Разработаны также дифференциальные потокомеры, в которых в качестве нагрева- 
телей и термометров используются токопроводящие пленки. В любом случае, измерение сводится к аналоговым измерениям резистивных термометров. Потокомеры-анемометры имеют более низкую точность и более длительное время отклика. Такие потокомеры широко используются в автомобильной промышленности. Подробное описание различных технологий измерения газовых потоков, включая сравнение их преимуществ и недостатков, а также информацию об их технических характеристиках см. в работах [2-5]. Всем этим методам присущи свои достоинства и недостатки, поэтому актуальной остается задача разработки новых типов потокомеров, направленных на расширение области их применения, повышение точности и увеличения их стабильности.

В данной работе предлагается новый принцип действия потокомеров, который основывается на измерении искажений теплового поля вокруг нагревателя при потоке. Температура таких искажений измеряется с помощью вибрирующих струн, играющих роль малоинерционных прецизионных термометров.

\section{2. Принцип работы потокомеров на основе вибрирующей струны}

Идея использования вибрирующих струн в качестве прецизионных термометров была предложена для диагностики пучков в ускорителях [6-9]. Такие приборы для измерения профилей пучков заряженных частиц/излучения/нейтронов мы называем мониторами вибрирующих струн (MBC). Принцип работы таких мониторов основан на большой чувствительности частоты колебаний струны, защемленной с концов, к ее натяжению, которое, в свою очередь, определяется температурой струны. Пучки заряженных частиц или излучения, падающие на струну, оставляют часть энергии в материале струны, что приводит к ее нагреву и, следовательно, изменению частоты ее собственных колебаний.

Возбуждение собственных колебаний производится при помощи магнитного поля, обеспечивающего взаимодействие тока, протекающего через струну с полем. Струна включена на положительный вход операционного усилителя. Такая положительная обратная связь приводит к усилению случайных флуктуаций струны, из которых выживают колебания на собственной частоте, так как добротность именно таких колебаний максимальна (подробнее о схеме автогенерации собственных колебаний см. в работе [9]).

Чувствительность струны (безразмерный коэффициент, связывающий относительное изменение частоты с относительным изменением длины струны) определяется по формуле

$$
\frac{\Delta F}{F}=\frac{\Delta \sigma}{2 \sigma}=\frac{E}{2 \sigma} \frac{\Delta L}{L}=\frac{E}{2 \sigma} \alpha \Delta T,
$$

где $F$ - начальная частота струны при начальной температуре, $\Delta F-$ изменение частоты струны, вызванное перегревом струны на величину $\Delta T, E$ - модуль упругости материала струны, $\sigma$ - начальное напряжение струны, $\alpha-$ коэффициент теплового расширения материала струны (предполагается, что измеряемый объект воздействует только на струну - это предположение близко к 
реальности для пучков заряженных частиц/излучения). Обычно начальное напряжение струны (напряжение струны при сборке) составляет 50-70\% от предела упругости материала. Безразмерное отношение $E / 2 \sigma$ составляет несколько сотен. Следует отметить, что чувствительность термометров сопротивления не содержит такого коэффициента умножения и, следовательно, значительно ниже.

Типичные частоты мониторов находятся в диапазоне 2-8 кГц, а стабильность частоты в течение нескольких часов составляет $\sim 0.005$ Гц. Соответствующая относительная точность составляет несколько единиц, умноженных на $10^{-6}$. Эта величина позволяла измерять интенсивность пучков/излучений, эквивалентных повышению температуры струны на доли мК (для струн из: нержавеющей стали - 0.3 мК, бронзы - 0.6 мК, вольфрама - 1.0 мК). Фактически вибрирующие струны представляются прецизионными термометрами с хорошей точностью и широким динамическим диапазоном. На рис.1 приведены графики зависимости частот струны из различных материалов от ее перегрева.

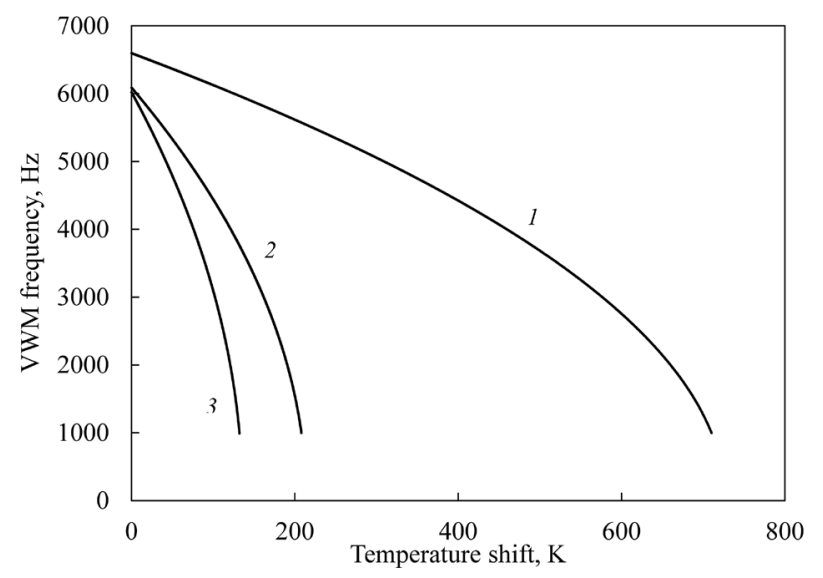

Рис.1. Зависимость частоты вибрирующей струны от перегрева по отношению к температуре сборки для струн из: 1 - вольфрама, 2 - бериллиевой бронзы (Beryllium Bronze - Cu97.5/Be2/Co-Ni0.5, UNS C17200), 3 - нержавеющей стали (AISI 316).

Как видим диапазон перегрева для вольфрамовой струны простирается до $\sim 700$ К, для бронзовой $>200$ К и для струн из нержавеющей стали - до температур 130 К. Подчеркнем, что речь идет именно о перегреве, в предположении, что струнный резонатор собирался при комнатной температуре, причем при сборке струна натягивается на 70\% от предела прочности материала. Случай, когда струна натягивается слабо и в дальнейшем охлаждается также возможен, однако, в данной работе не рассматривается.

Отметим, что попытки измерения газовых потоков с помощью МВС были предприняты еще в 2002 г. [10]. Для повышения чувствительности частоты струны по отношению к ее обдуву газом была разработана специальная схема, когда помимо возбуждающего колебания на собственной частоте тока, на струну 


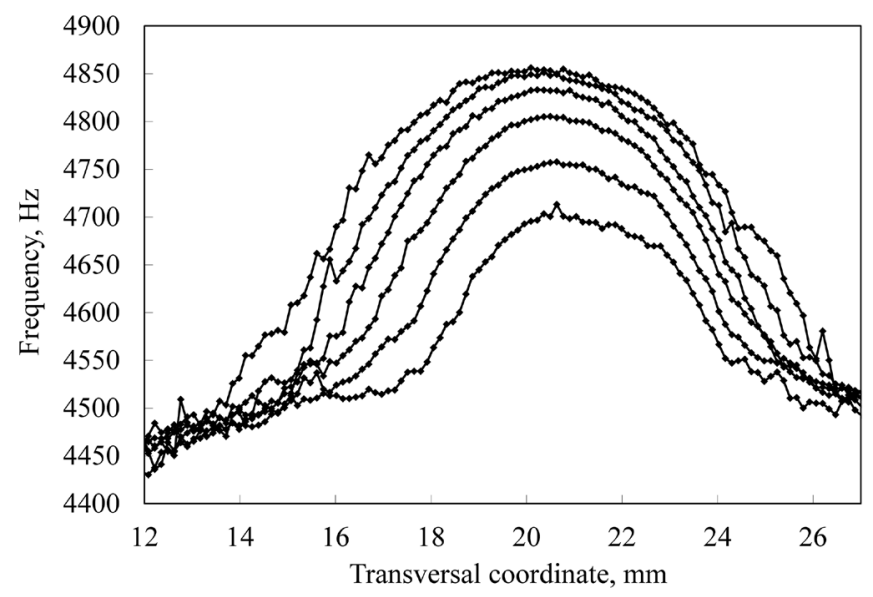

Рис.2. Поперечные профили струн в различных ее ортогональных сечениях, расположенных на расстояниях 4 мм друг от друга (линии профилей расположены снизу вверх, в порядке увеличения расстояния от сопла, создающего струю).

одновременно подавался также постоянный ток. Такой монитор позволил произвести сканирование сечений широких газовых струй. Струна в зависимости от локального потока обдува охлаждалась, что приводило к увеличению ее частоты. На рис.2 изображены профили в различных ортогональных сечениях струн на расстоянии 4 мм друг от друга.

Эксперименты проводились также с продувкой газом (воздухом) через апертурное отверстие МВC, разработанного для измерения профиля ондуляторного излучения источника синхротронного излучения APS (Argonne Lab, USA) [11]. Во всех случаях использовались мониторы, предназначенные для профилирования пучков заряженных частиц. Апертура таких мониторов не имела специализированной газодинамической структуры и не обеспечивала эффективного взаимодействия газовых потоков со струной.

\section{3. Разработка модели потокомера}

Для потокомеров на основе вибрирующих струн был разработан специальный резонатор, содержащий несколько струн (две струны-термометра и струнунагреватель) и канал, обеспечивающий эффективное взаимодействие измеряемого потока с тепловым полем нагревателя. Измеряемый поток направляется в зазор магнитного поля ортогонально струнам, обеспечивая их обтекаемость по всей длине. В зазоре в одной плоскости помещаются три струны, средняя струна-нагреватель и по бокам - вибрирующие струны-термометры.

Общий вид резонатора представлен на рис.3. Измеряемый поток распространяется вдоль оси $Z$ (на рис.3 сверху вниз). Следуя принятой в метеорологии терминологии вибрирующую струну, на которую поток падает непосредственно (на рис.3 - верхнюю), назовем наветренной, а на нижнюю струну, куда сносится поток тепла от нагревателя, назовем подветренной [12]. Для струн и нагревателя 


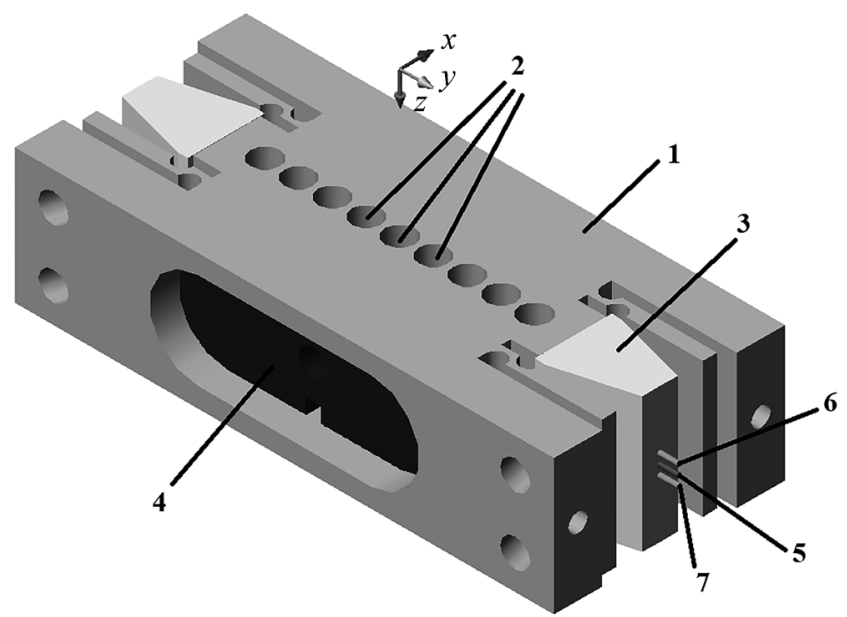

Рис.3. Изометрический вид резонатора потокомера на основе вибрирующих струн. 1 - корпус резонатора (нержавеющая сталь/латунь), 2 -приемные отверстия, 3 - керамические клинья, 4 - магнитные полюса системы возбуждения собственных колебаний струн-термометров, 5 - нагревательная струна, 6 - наветренная струна-термометр (струна, ближняя к падающему на резонатор потоку), 7 - подветренная струнатермометр (струна, расположенная за нагревателем).

использовалась проволока из прошедшей специальную термообработку нержавеющей стали диаметром 100 мкм. Все струны зажаты между клиновидными керамическими пластинами. Магнитная система состоит из четырех постоянных цилиндрических магнитов, замкнутых магнитными полюсами. Измеряемый поток по системе специальных каналов в корпусе потокомера (не изображен) подводится к приемным отверстиям резонатора.

Измеряемый поток воздуха распространяется вдоль оси $Z$ и входит в приемные отверстия на торце корпуса потокомера:

На рис.4 изображено сечение резонатора плоскостью, проходящей через три струны (нагреватель и две вибрирующих).

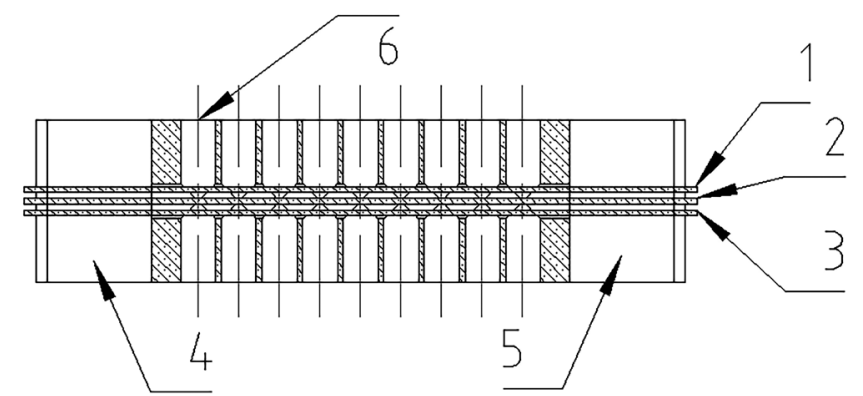

Рис.4. Сечение резонатора плоскостью, в которой расположены струны. Предполагается, что измеряемый поток распространяется сверху вниз: 1 - наветренная струна, 2 - нагреватель, 3 - подветренная струна, 4 и 5 - плоскости керамических клиньев, 6 - ряд приемных отверстий потока на торце корпуса потокомера. 
При заданной геометрии потокомера (длина и диаметр струн, их взаимное расположение по отношению к нагревателю), важным параметром является выбор материала струн. Для нагревательной струны хорошим вариантом является нержавеющая сталь с большим удельным сопротивлением. Тот же материал может использоваться и для самих вибрирующих струн. Выбор одинакового материала для нагревательной и вибрирующих струн упрощает сборку монитора, поскольку зажим концов всех трех струн производится одной парой керамических клиньев.

\section{4. Экспериментальные результаты, калибровка потокомера}

Для калибровки потокомеров требуется произвести ряд экспериментов, в которых при заданных значениях потока измеряется изменение частот струн-термометров для фиксированной величины тока через нагревательную струну.

Ток через нагревательную струну задавался с помощью стабилизированной схемы источника тока на базе микросхемы LM337.

Стабильные потоки газа (воздуха) создавались закачкой воздуха в ресивер с большим объемом под давлением около 5-6 бар и пропуском накопленного воздуха через тонкое отверстие вентиля. Измерение потока воздуха производилось с помощью газового барабанного счетчика ГСБ-400кл1, обладающего преимуществом абсолютных измерений. В эксперименте использовался двунаправленный вентиль, в одном положении, пропускающий поток через потокомер вибрирующей струны и в другом - в атмосферу. В положении вентиля, выпускающем поток в атмосферу, величина потока настраивалась с помощью прецизионного тонкого вентиля и измерением времени, требующемся для пропуска определенного объема воздуха через счетчик ГСБ. На рис.5 и 6 приведены результаты экспериментов для двух значений тока: 50.4 мА и 101 мА соответственно.

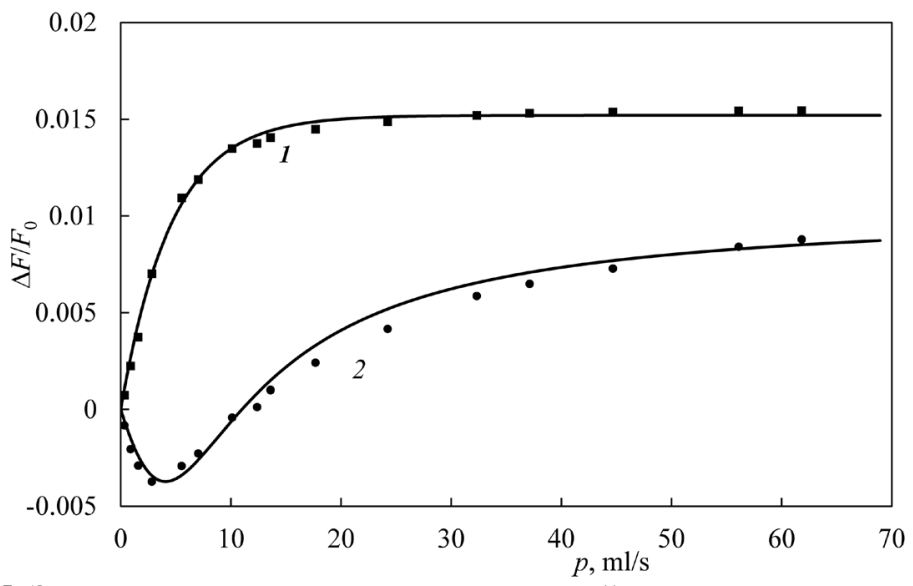

Рис.5. Зависимость относительных изменений частот струн-термометров от величины потока через потокомер при токе через нагревательную струну 50.4 мА. Кружочки - сигналы частоты с наветренной струны, 1 фитирование этих сигналов, квадратики - сигналы частоты с подветренной струны, 2 - фитирование этих сигналов. 


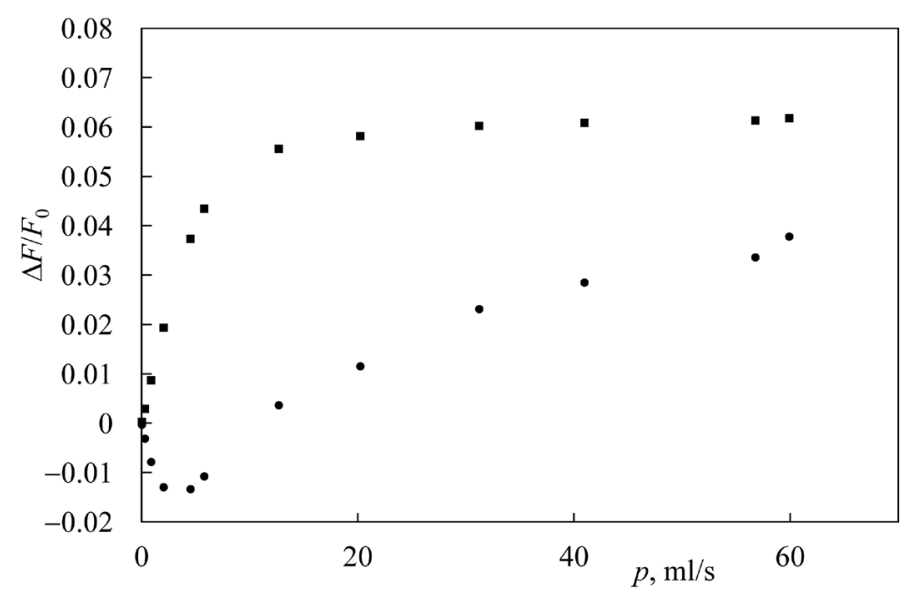

Рис.6. Зависимость относительных изменений частот струн-термометров от величины потока через потокомер при токе через нагревательную струну 101 мА. кружочки - сигналы частоты с наветренной струны, квадратики - сигналы частоты с подветренной струны.

Из рис.5 видно, что поведение частот струн существенно зависит от величины потока, так и от позиционирования струн относительно нагревателя (наветренная, подветренная). Весь диапазон потоков составлял от 0 до 70 мл/с. Наветренная струна, (на рис.5 отмеченная как первая) показывает достаточно крутой, но монотонный рост частоты при увеличении потока с выходом на предельную величину $~ 0.015$. Подветренная струна при малых потоках (до 3-4 мл/с) дополнительно прогревается сносимым от нагревателя тепловым потоком, и частота струны падает. Однако, при значениях потока > этой величины охлаждение струны измеряемым потоком превышает эффект сноса тепла от нагревателя, и частота струны начинает расти. Рост частоты при этом происходит гораздо медленнее, чем для наветренной струны и вплоть до величин потока до 62 мл/с (максимальное значение в данном эксперименте) выхода частоты на предельное значение не наблюдается. Похожую картину мы наблюдали также в эксперименте, когда ток через нагревательную струну составлял 101 мА (см. рис.6). При таком повышении тока в два раза тепловыделение на струне повышается в четыре раза, что приблизительно соответствует новому предельному значению относительного сдвига частоты наветренной струны 0.06. Диапазоны характерных значений потоков практически те же, поэтому в качестве рабочего тока через струну выберем значений тока 50.4 мА и именно данные рис.5 выберем для калибровки потокомера.

Очевидно, что при малых потоках $p$, когда изменения частот наветренной и подветренной струн имеют разный знак можно использовать дифференциальный метод, учитывающий также возможные тепловые дрейфы в случае длительных измерений. Для больших значений потоков следует учитывать сильную нелинейность частотных сигналов. Предлагается весь диапазон значений потоков разбить на три области: 
- диапазон малых потоков ( $p<3$ мл/с), в котором сигналы с обеих струн пропорциональны измеряемому потоку;

- диапазон реагирования наветренной струны (3 мл/с $<p<10$ мл/с), в этой области частота подветренной струны претерпевает экстремум и содержит неоднозначность;

- диапазон нелинейного реагирования наветренной струны ( $p>10$ мл/с), в этой области сигнал с наветренной струны выходит на предельное значение, однако подветренная струна продолжает сохранять чувствительность к потоку вплоть до значений 60-70 мл/с.

В качестве индикатора выбора диапазона будем использовать показания наветренной струны. Вначале для каждого из указанных диапазонов мы использовали набор соответствующих экспериментальных данных с линейной регрессией по показаниям обоих струн для первого диапазона, и квадратичной регрессии для второго и третьего диапазонов соответственно по показаниям наветренной и подветренной струн. Однако, при таком подходе обнаружилась достаточно сильная нестыковка значений ( $10 \%)$ на границах диапазонов. Поэтому мы разработали метод фитирования экспериментальных данных одной функцией по всему диапазону значений. Для показаний наветренной струны хороший результат дало использование функции

$$
\Delta F_{1} / F_{10}=A(1-\exp (-p / B)),
$$

где $p$ - значение потока, выраженное в мл/с. Для величин $A=0.015$ и $B=4.6$ величина среднеквадратичного отклонения аналитической кривой от экспериментальных значений составляет $1 \times 10^{-7}$, что вполне приемлемо. Для показаний подветренной струны мы использовали дробную функцию вида

$$
\Delta F_{2} / F_{20}=\frac{-a p+b p^{2}}{c+d p^{2}} .
$$

Значения $a=0.011, b=0.001, c=6, d=0.095$ дают среднеквадратичное отклонение кривой (2) от экспериментальных точек порядка $2.6 \times 10^{-7}$.

Для использования формул (1) и (2) их следует обратить относительно величины потока $p$. Для формулы (2) имеем:

$$
p=B \ln \left(\frac{A}{A-\Delta_{1}}\right),
$$

где $\Delta_{1}=\Delta F_{1} / F_{10}$.

Обращение формулы (3) требует решения квадратного уравнения, таким образом вся конструкция записывается в виде

$$
p_{ \pm}=\frac{a \pm \sqrt{a^{2}+4 \Delta_{2} c\left(b-d \Delta_{2}\right)}}{2\left(b-d \Delta_{2}\right)},
$$

где $\Delta_{2}=\Delta F_{2} / F_{20}$.

Графики уравнений (4) и (5) представлены на рис.7.

На рис.8 приведены диапазоны разбивки сигналов с вибрирующих струн, 


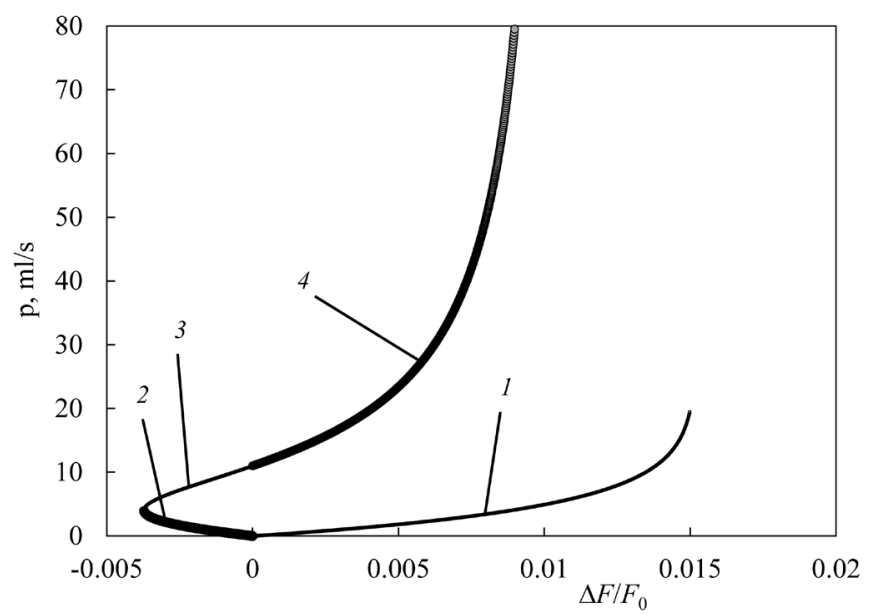

Рис.7. Аналитические калибровочные функции: 1 - функция калибровки наветренной струны (формула (4)); 2, 3, 4- функция калибровки подветренной струны (2 и 3 - корни уравнения (5) со знаками $(-)$ и (+) для отрицательных значений $\Delta_{2}, 4$ - корень уравнения (5) со знаком плюс для положительных значений $\Delta_{2}$ ).

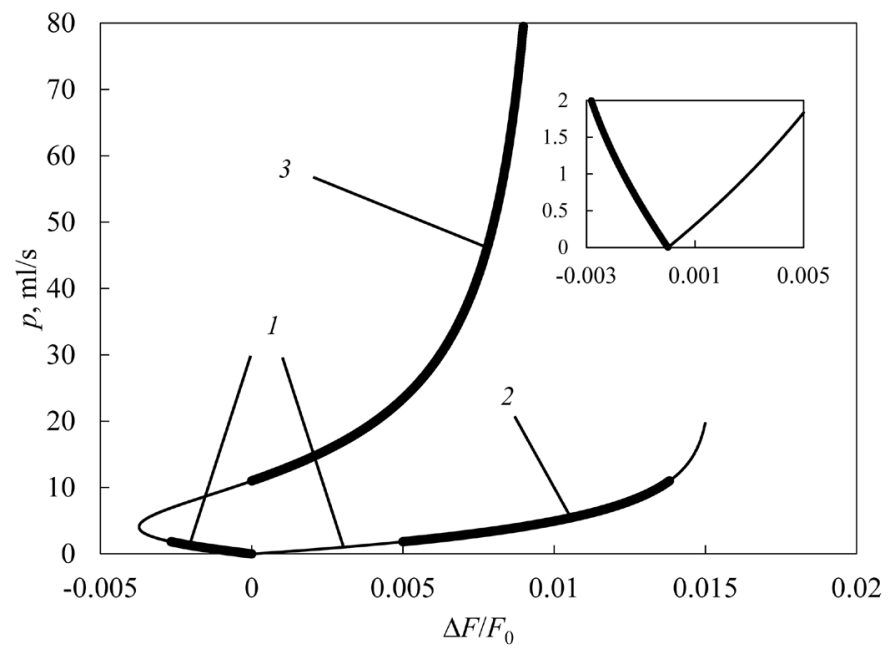

Рис.8. Разбивка калибровочных данных на три характерных диапазона: 1 - область линейных откликов для обеих измерительных струн, 2 и 3 области характерного изменения частоты наветренной и подветренной струн соответственно. На вставке область 1 в увеличенном масштабе.

которые сшиваются по построению (применяются функции с носителем по всему диапазону значений частот/потоков).

Итак, объединяя полученные результаты, получим следующие формулы для трех характерных диапазонов частотных откликов:

Область линейных откликов обоих вибрирующих струн (диапазон 1, $0<\Delta_{1}<0.005$ ) 


$$
p=\frac{1}{2} B \ln \left(\frac{A}{A-\Delta_{1}}\right)-\frac{1}{2} \frac{a-\sqrt{a^{2}+4 \Delta_{2} c\left(b-d \Delta_{2}\right)}}{2\left(b-d \Delta_{2}\right)},
$$

(отмеченный выше дифференциальный метод соответствует разложению этой формулы по малым величинам $\Delta_{1}$ и $\Delta_{2}$ );

Область характерного изменения частоты наветренной струны (диапазон 2 , $\left.0.005<\Delta_{1}<0.0138\right)$

$$
p=B \ln \left(\frac{A}{A-\Delta_{1}}\right) ;
$$

Область характерного изменения частоты подветренной струны (диапазон 3 , $\left.0.0138<\Delta_{1}\right)$ :

$$
p=\frac{-a+\sqrt{a^{2}+4 \Delta_{2} c\left(b-d \Delta_{2}\right)}}{2\left(b-d \Delta_{2}\right)} .
$$

\section{5. Потокомер в качестве акселерометра}

Интересно отметить, что искажение теплового поля может происходить также в результате резкого движения потокомера. Этот эффект был предложен для разработки акселерометров, не содержащих твердотельную инертную массу (см., например, обзор по теме [13]). В оригинальной работе [14], которую цитируют как первую с предложением использования зависимости теплопереноса от ускорения, предложено использовать в качестве нагревателя консольно-закреплённую пластину с возможностью движения (см. также работу [15). Полный отказ от каких-либо твердых тел и переход только на газовую среду, по-видимому, был сформулирован в патенте [16] (см. также работу [17]). Предложение включало два температурно-чувствительных элемента, установленных в герметичном корпусе, содержащем газ. Источник тепла в газе создает конвективные потоки, измеряемые термометрами. Приложенное к корпусу линейное ускорение изменяет конвективные потоки, вызывая разность температур между термочувствительными элементами. Разность температур измеряется как разница в электрическом сопротивлении между двумя термочувствительными элементами, которая пропорциональна ускорению. Экспериментальная демонстрация метода была показана в работе [18]. Типичная одноосная конструкция содержит три струны в одной плоскости (при микромашинном изготовлении с размерами 1мм), одна из которых служит нагревателем, а две струны по бокам - термометрами (см. также работу [19]).

Разработанная нами конструкция потокомера практически без переделок была применена в качестве акселерометра без инертной массы. Потокомер-акселерометр был расположен на платформе, подвешенной на пружине. Ось ускорения направлена в плоскости струн перпендикулярно их направлению (см. рис.9).

Результаты измерений частот потокомера представлены на рис.10. Входные/выходные отверстия на потокомере были открыты на воздух. Начальные 


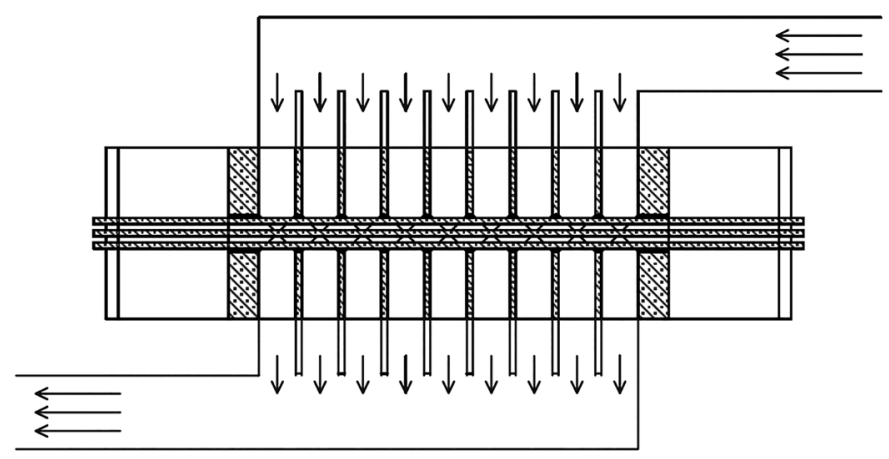

Рис.9. Использование потокомера в качестве акселерометра. Потоки воздуха при ускорении потокомера наверх показаны стрелками.

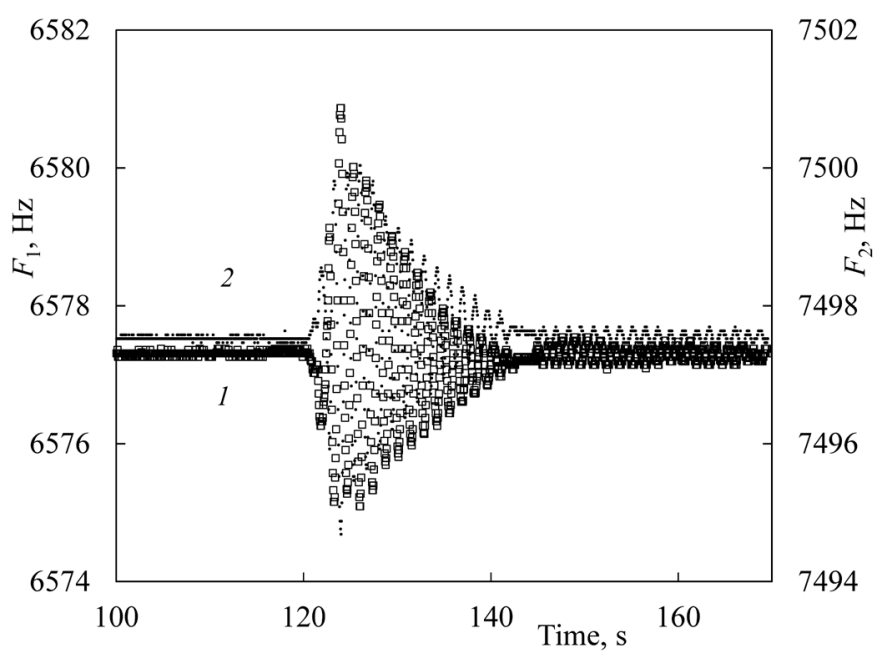

Рис.10. Данные частот вибрирующих струн потокомера, совершающего колебания на пружинном подвесе: 1 и 2 - сигналы с наветренной (квадратики) и подветренной (точечки) струн.

значения частот (6577.3 Гц и 7497.5 Гц) позволяли производить их достаточно быстрые измерения в течение времени 50.4 мс.

Видно, что смещения частот имеют разную направленность, это значит, что измеряемые потоки находятся в линейной области отклика (область 1 на рис.8).

Ускорение приводится в единицах ускорения свободного падения (абсолютные значения оценены по порядку величины, на основе измерения максимального смещения потокомера от положения равновесия).

\section{6. Заключение}

Разработаны и изготовлены потокомеры на базе вибрирующих струн, получены первые экспериментальные результаты (см. рис 11). Произведен подробный анализ калибровочных экспериментов и разработан алгоритм вычисления потоков по результатам нелинейных сигналов частоты в диапазоне потоков 


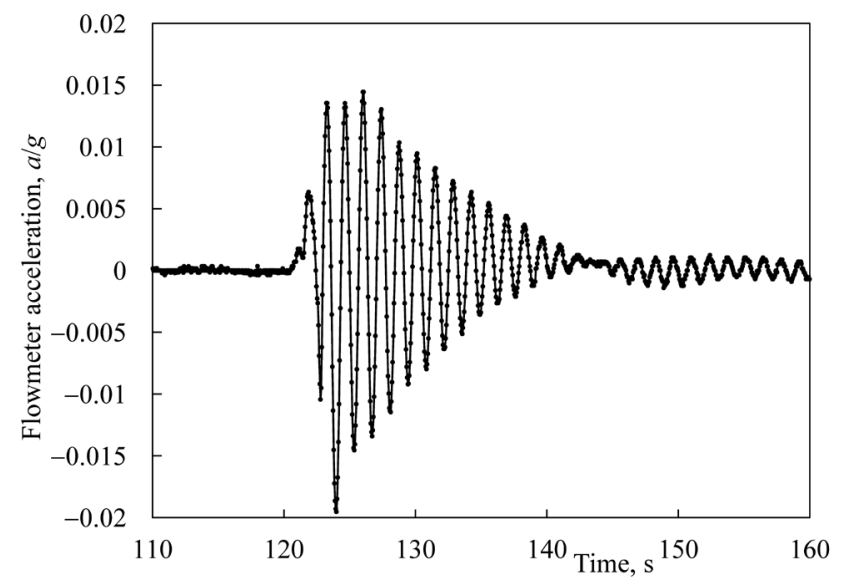

Рис.11. Вычисленное по результатам эксперимента ускорение корпуса потокомера (в единицах ускорения свободного падения).

приблизительно в двадцать раз превышающих диапазон линейных откликов наветренной и подветренной струн. Потокомер использовался также в качестве акселерометра, здесь также получены первые обнадеживающие результаты.

Разработанный прибор, оснащенный средствами визуализации измерений и интерфейсом с компьютером, может иметь широкий спектр использования в различных областях науки и техники, в том числе и для спирометрических измерений в медицине.

Исследование выполнено при финансовой поддержке Комитета по науке РА в рамках научного проекта 20APP-2G001.

\section{ЛИТЕРАТУРА}

1. https://portal.endress.com/wa001/dla/5000581/9936/000/01/FA00005D.pdf

2. И.И. Билинский, М.А. Стасюк, М.В. Гладышевский. Вісник Національного технічного університету «ХПІ», № 42, (2015).

3. R.C. Baker. Flow Measurement Handbook. Cambridge University Press, CB2 2RU, UK (2000).

4. R. Thorn. Basic Principles of Flow Measurement, John Wiley \& Sons, Ltd. ISBN: 0-47002143-8, 2005.

5. R. Thorn, A. Melling, et al. Flow Measurement, in: Measurement, Instrumentation, and Sensors Handbook, Electromagnetic, Optical, Radiation, Chemical, and Biomedical Measurement, John G. Webster, Halit Eren, CRC Press. (2017).

6. S.G. Arutunian, N.M. Dobrovolski, M.R. Mailian, I.G. Sinenko, I.E. Vasiniuk. Phys. Rev. Spec. Top.-Accel. Beams, 2, 122801, (1999).

7. S.G. Arutunian, M.R. Mailian, K. Wittenburg. NIM A, 572, 1022 (2007).

8. S.G. Arutunian, J. Bergoz, M. Chung, G.S. Harutyunyan, E.G. Lazareva. NIM A, 797, $37(2015)$.

9. S.G. Arutunian, A.V. Margaryan, G.S. Harutyunyan, E.G. Lazareva, M. Chung, D. Kwak, D.S. Gyulamiryan. JINST, 16, R01001 (2021). 
10. S.G. Arutunian, A.E. Avetisyan, N.M. Dobrovolski, M.R. Mailian, I.E. Vasiniuk, K. Wittenburg, R. Reetz. EPAC-8 (June 2002, Paris, France), p. 1837, 2002.

11. G. Decker et al. DIPAC, p 36 (2007).

12. https://meteoinfo.ru/glossary/6915-2013-05-07-10-26-22

13. R. Mukherjee, J. Basu, P. Mandal, P.K. Guha. Journal of Micromechanics and Microengineering, 27, 123002 (2017).

14. R. Hiratsuka, D.C. van Duyn, T. Otaredian, P. de Vries. TRANSDUCERS'91 (San Francisco, CA, USA, 1991), p. 420 (1991).

15. R. Hiratsuka, D. C. van Duyn, T. Otaredian, P. de Vries, P. M. Sarro. Sensors and Actuators A, 32, 380 (1992).

16. R. Dao et al. Convective Accelerometer and Inclinometer, United States Patent 5581034, (1996).

17. M. Gaitan et al. Method of Manufacture of Convective Accelerometers, United States Patent US 6171880 B1, (2001).

18. A.M. Leung, J. Jones, E. Czyzewska, J. Chen, M. Pascal. Micromachined accelerometer with no proof mass, IEDM Technical Digest, p. 899, (1997).

19. F. Mailly, A. Giani, A. Boyer. Micromachined Thermal Accelerometer without Proof Mass. Springer, Boston: MA (2006).

GAS FLOWMETER BASED ON VIBRATING WIRES

\author{
M.A. AGINIAN, S.G. ARUTUNIAN, G.S. HARUTYUNYAN, E.E. GUKASYAN, \\ E.G. LAZAREVA, A.V. MARGARYAN, L.A. POGHOSYAN, \\ M. CHUNG, D. KWAK, R. REETZ
}

This paper proposes a new type of gas flowmeters based on measurements of deformations of the heater's thermal field in the presence of flow by using vibrating wires. The advantage of such flowmeters as compared to the known ones using a similar thermal principle is the improved accuracy of temperature measurements as compared to resistance thermometers or thermocouples. The use of thin wires also makes it possible to increase the speed of the flowmeters. The use of $20-30 \mathrm{~mm}$ long wires also makes it possible to create wide-aperture receiving inlets for gas flows. A natural property of the developed flowmeters is also their twodirectionality. A sample of flowmeters based on vibrating wires was made, and calibration experiments were carried out. An experiment in which the flowmeter was used to measure acceleration was performed. 